\title{
Re-resection in Incidental Gallbladder Cancer: Survival and the Incidence of Residual Disease
}

\author{
Elise A. J. de Savornin Lohman, MD, PhD ${ }^{1}$, Lydia G. van der Geest, PhD $^{2}$, Tessa J. J. de Bitter, MSc ${ }^{3}$, \\ Iris D. Nagtegaal, $\mathrm{MD}, \mathrm{PhD}^{3}$, Cornelis J. H. M. van Laarhoven, $\mathrm{MD}, \mathbf{P h D}^{1}$, Peter van den Boezem, MD, $\mathbf{P h D}^{1}$, \\ Chella S. van der Post, MD, PhD $^{3}$, and Philip R. de Reuver, MD, PhD ${ }^{1}$ \\ ${ }^{1}$ Department of Surgery, Route 618, Radboudumc, Nijmegen, The Netherlands; ${ }^{2}$ Netherlands Comprehensive Cancer \\ Organization, Utrecht, The Netherlands; ${ }^{3}$ Department of Pathology, Radboudumc, Nijmegen, The Netherlands
}

\begin{abstract}
Background. Re-resection for incidental gallbladder cancer (iGBC) is associated with improved survival but little is known about residual disease (RD) and prognostic factors. In this study, survival after re-resection, $\mathrm{RD}$, and prognostic factors are analyzed.

Methods. Patients with iGBC were identified from the Netherlands Cancer Registry, and pathology reports of reresected patients were reviewed. Survival and prognostic factors were analyzed.

Results. Overall, 463 patients were included; 24\% $(n=110)$ underwent re-resection after a median interval of 66 days. RD was present in $35 \%$ of patients and was most frequently found in the lymph nodes $(23 \%)$. R0 resection was achieved in 93 patients (92\%). Median overall survival (OS) of patients without re-resection was 13.7 (95\% confidence interval [CI] 11.6-15.6), compared with 52.6 months (95\% CI 36.3-68.8) in re-resected patients $(p<0.001)$. After re-resection, median OS was superior in patients without RD versus patients with RD (not reached vs. 23.1 months; $p<0.001$ ). In patients who underwent reresection, $\mathrm{RD}$ in the liver (hazard ratio [HR] 5.54; $p<0.001$ ) and lymph nodes (HR 2.35; $p=0.005$ ) were the only significant prognostic factors in multivariable
\end{abstract}

Electronic supplementary material The online version of this article (https://doi.org/10.1245/s10434-019-08074-4) contains supplementary material, which is available to authorized users.

(C) The Author(s) 2019

First Received: 23 July 2019; Published Online: 18 November 2019

P. R. de Reuver, MD, PhD

e-mail: philip.dereuver@radboudumc.nl analysis. Predictive factors for the presence of $\mathrm{RD}$ were pT3 stage (HR 25.3; $p=0.003$ ) and pN1 stage (HR 23.0; $p=0.022$ ).

Conclusion. Re-resection for $\mathrm{iGBC}$ is associated with improved survival but remains infrequently used and is often performed after the optimal timing interval. $\mathrm{RD}$ is the only significant prognostic factor for survival after re-resection and can be predicted by $\mathrm{pT}$ and $\mathrm{pN}$ stages.

Gallbladder cancer (GBC) is the most prevalent biliary tract malignancy and the sixth most common gastrointestinal malignancy worldwide. ${ }^{1}$ Due to an asymptomatic course in the early stages, patients are frequently diagnosed in an advanced stage and prognosis is extremely poor. ${ }^{2-5}$ However, long-term survival does occur in patients with early-stage tumors. ${ }^{6}$ These patients are most frequently diagnosed incidentally (iGBC), after cholecystectomy for presumed benign gallbladder disease. ${ }^{7-9}$ Due to the growing number of laparoscopic cholecystectomies performed, iGBC is an increasingly relevant clinical issue. ${ }^{10,11}$ Noticeably, especially in the Western world, many GBCs are detected as an incidental finding. ${ }^{2,12}$

In order to prevent early locoregional recurrence, reexploration and definitive resection is currently recommended for patients with tumors invading the muscle layer and no evidence of disseminated disease. ${ }^{10}$ Re-resection involves a partial hepatectomy of segments $4 \mathrm{~b} / 5$, either as a full segmentectomy or wedge excision, and resection of the hepatoduodenal lymph nodes. ${ }^{13}$

Re-resection is associated with improved survival in retrospective studies. However, it is still controversial whether resecting residual disease (RD) actually improves 
survival or whether it merely enables more complete staging and consequently provides more accurate estimation of survival.

Prognosis after re-resection appears to be primarily determined by the presence of RD and lymph node metastases. ${ }^{6,14-16}$ Interestingly, although the likelihood of detecting RD increases concurrently with $\mathrm{T}$ stage, a study including 135 patients found that survival did not differ between T2 and T3 tumors in patients in whom no RD was detected. ${ }^{15}$ This finding suggests that rather than $\mathrm{T}$ stage, the presence of RD after re-resection appears to be the primary predictor for survival.

Evidently, identifying patients at risk for RD after reresection could greatly improve candidate selection for additional surgery. Patients who are likely to have RD could potentially benefit from more aggressive surgery. On the other hand, in patients at low risk of $\mathrm{RD}$, a more conservative approach could be justified.

The aim of this study was to assess survival of iGBC patients with and without re-resection. Second, we assessed the prognostic value of histopathological characteristics on survival after re-resection.

\section{METHODS}

This was a retrospective, nationwide cohort study that was approved by the NCR Ethical Review Board. A waiver for ethical approval was provided by the Medical Ethics Review Committee of the Arnhem-Nijmegen region (CMO A-N, nr. 2017-3912), and the Strengthening the Reporting of Observational Studies in Epidemiology (STROBE) statement for reporting of observational cohort studies was followed. ${ }^{17}$

\section{Patient Selection and Variable Definitions}

All patients diagnosed with iGBC from 2000 to 2016 were identified from the Netherlands Cancer Registry (NCR). The NCR contains data on all newly diagnosed malignancies, including year of diagnosis, patient age and gender, and tumor characteristics (cTNM and pTNM stage). ${ }^{18}$ Notification sources were the nationwide network and registry of histopathology and cytopathology in The Netherlands (PALGA ${ }^{19}$ ) and data from the National Registry of Hospital Discharge Diagnoses. Follow-up data on vital status (complete until February 2018) were provided by linkage to the automated Municipal Personal Records Database. iGBC was defined as GBC diagnosed based on postoperative histopathological examination. All patients with pre- or perioperative suspicion of GBC (defined as suspicion of $\mathrm{GBC}$ on preoperative imaging or findings suspect for malignancy during surgery) were excluded since the NCR categorizes these patients as suspected GBC. Patients with T1a disease or metastatic disease (detected by imaging during postoperative re-staging or during re-exploration within 6 months of diagnosis) were excluded from analysis since these patients have no indication for additional radical surgery.

Re-resection was defined as any additional, GBC-directed surgery within 6 months after the primary surgery. A retrospective review of the complete pathology reports of re-resected patients was performed using data supplied by PALGA. For patients who received a re-resection, the pTNM stage as reported after primary surgery was used to reconstruct the initial TNM stage. Because the location of the tumor was frequently not reported, no differentiation between serosal and liver side tumors could be made, and all tumors were classified according to the 7th edition of the American Joint Committee on Cancer (AJCC) staging manual. ${ }^{18}$ Adjuvant chemo(radio)therapy is not considered standard of care in The Netherlands and was not administered to any of the patients throughout the study period.

For re-resected patients for whom complete histopathological reports were available, the following variables were extracted from the primary surgery report: type of surgery (laparoscopic cholecystectomy, open cholecystectomy, other, unspecified), pTNM stage, tumor size, tumor differentiation, presence of perineural/perivascular/lymphatic growth, and radicality (R0 defined as no microscopically present tumor $<1 \mathrm{~mm}$ from the resection margin). The following variables were assessed in the reresection report: cystic duct stump resection (yes/no), lymphadenectomy (yes [number of lymph nodes resected]/ no), liver resection (no/gallbladder bed/one segment/two segments $/ \geq 3$ segments), presence and location of RD (defined as findings of microscopic liver/lymph node/cystic duct involvement in the pathological examination after radical surgery) and radicality of the re-resection.

\section{Statistical Analysis}

Patient and tumor characteristics were described using counts and percentages for categorical variables, and means and ranges for continuous variables. Patients who underwent re-resection were categorized as having $\mathrm{T} 1 \mathrm{~b}$, $\mathrm{T} 2$, or $\mathrm{T} 3 / \mathrm{Tx}$ disease, based on the $\mathrm{T}$ stage after primary resection (no patients with $\mathrm{T} 4$ disease received a re-resection). All analyses for patients with a re-resection were conducted using the $\mathrm{T}$ stage as assessed after the primary resection. The Chi square or Fisher's exact tests were used, where appropriate, to assess differences in the extent of reresection performed and the presence and location of $\mathrm{RD}$, and Kaplan-Meier curves were used to calculate the median survival times. Survival was defined as the time in days from the date of diagnosis (primary surgery) until the 
date of death from any cause or the date of end of followup. ${ }^{20}$ Log-rank testing and Cox regression analysis were used to compare survival between groups of patients. To deal with immortal time bias of patients who underwent reresection, patients with a follow-up duration of $<90$ days after resection were excluded for all comparative survival analyses. Additionally, to reduce treatment selection bias in the calculation of median survival times, the Kaplan-Meier method was repeated in patients under 65 years of age.

Cox regression analysis was used to calculate hazard ratios (HRs) for potential prognostic factors in patients who underwent re-resection, and logistic regression was used to identify factors predictive for RD. Covariates were selected based on the literature and were entered in the multivariable model when statistically relevant $(p<0.1)$ on univariable analysis. A stepwise forward selection approach was used. Missing data were determined to be 'missing at random' (unrelated to the outcome, potentially related to other parameters) and complete case analysis was used to assess covariates. ${ }^{20,21}$

$p$ values $<0.05$ were considered statistically significant, and all tests of significance were two-tailed. Statistical analyses were conducted using the SPSS 25.0 statistical package (IBM Corporation, Armonk, NY, USA).

\section{RESULTS}

\section{Patient and Tumor Characteristics}

A total of 463 patients with iGBC were included (Fig. 1), of whom 110 patients (23\%) underwent re-resection. Patient and tumor characteristics are displayed in Table 1. Patients with a re-resection were significantly younger; the mean age difference was 10 years and $43 \%$ of patients $\leq 65$ years of age received a re-resection, as opposed to $15 \%$ of patients aged 66 years or older $(p<0.001)$. Furthermore, re-resected patients were more likely to have T2 disease $(66 \%$ vs. $49 \% ; p=0.020)$ and node-positive disease ( $12 \%$ vs. $6 \% ; p=0.001)$.

\section{Re-resection Procedures and Histopathology \\ Assessment}

Complete histopathology reports were available in 102 patients who underwent re-resection. Primary surgery of these 102 patients was laparoscopic cholecystectomy in 26 (25\%) patients, open cholecystectomy in $4(4 \%)$ patients, subtotal cholecystectomy in $6(6 \%)$ patients, and unspecified in $66(65 \%)$ patients. The median interval between primary surgery and re-resection was 66 days [interquartile range (IQR) 47-83]. An overview of the re-resection procedures conducted and the incidence of RD is provided in
Fig. 2. Ninety-seven patients underwent dissection of the hepatoduodenal ligament, with a median lymph node harvest of 3 (range 2-20). Seventy-three patients underwent resection of the liver parenchyma; gallbladder bed resection in $55(75 \%)$ patients, gallbladder bed resection plus resection of segments 4 and 5 in $17(23 \%)$ patients, and right hemihepatectomy in $1(1 \%)$ patient. Fifty-three $(52 \%)$ patients underwent resection of the cystic duct stump, of whom $8(8 \%)$ also underwent extrahepatic bile duct resection.

No significant differences in the extent of resection was found between $\mathrm{T}$ stages (Fig. 2). RD was significantly more present in re-resection specimens of patients with $\mathrm{T} 3$ disease. R0 re-resection was achieved in $92 \%$ of patients across the re-resected cohort, but in only $72 \%$ of patients with T3 disease $(p<0.001)$.

\section{Survival in Incidental Gallbladder Cancer}

The median follow-up in the entire cohort $(n=463)$ was 17.8 months (IQR 8.1-36.7), and median overall survival (OS) was 18.3 months $[95 \%$ confidence interval (CI) 14.1-22.4]. Median OS of iGBC patients without re-resection was 13.7 (95\% CI 11.6-15.6), compared with 52.6 months (95\% CI 36.3-68.8) in patients who underwent re-resection $(p<0.001)$. When patients with a follow-up duration of $<90$ days from the primary surgery were excluded from the analysis, survival was 16.1 months (95\% CI 13.7-18.5) in patients without re-resection and 56.3 months (95\% CI 49.0-63.5) in re-resected patients $(p<0.001)$ (Fig. 3a). When selecting patients under the age of 65 years and with a follow-up duration of $\geq 90$ days, re-resection was still associated with superior survival (18 vs. 77 months; $p<0.001$ ).

In multivariable analysis including patients with $\geq 90$ days of follow-up, and controlling for age, $\mathrm{T}$ stage, nodal status, resection margin and tumor grade, re-resection remained a significant predictor for superior survival (HR 0.464 , 95\% CI $0.338-0.639 ; p<0.001$ ) [electronic supplementary Table 1].

In a subgroup analysis of all patients who had tumorfree resection margins at the primary resection $(N=226)$ median OS was 25.9 months in patients without re-resection (95\% CI 14.3-37.5) versus 83.8 months (95\% CI 41.6-125.9) in patients who received a re-resection ( $p<0.001)$. After excluding patients with $<90$ days of follow-up, this difference persisted: 28.2 months (R0) versus 90.0 months (R1) $(p<0.001)$. Median OS (after exclusion of patients with a follow-up duration of $<90$ days) in re-resected $\mathrm{T} 2$ disease was 60.0 months, versus 18.1 months $(p<0.001)$ in non-re-resected T2 disease. Median OS in re-resected T3 disease was 23.1 months versus 12.1 months $(p<0.015)$ in non-re-resected T3 
FIG. 1 Selection of the included patients. $G B C$ gallbladder cancer, $i G B C$ incidental gallbladder cancer

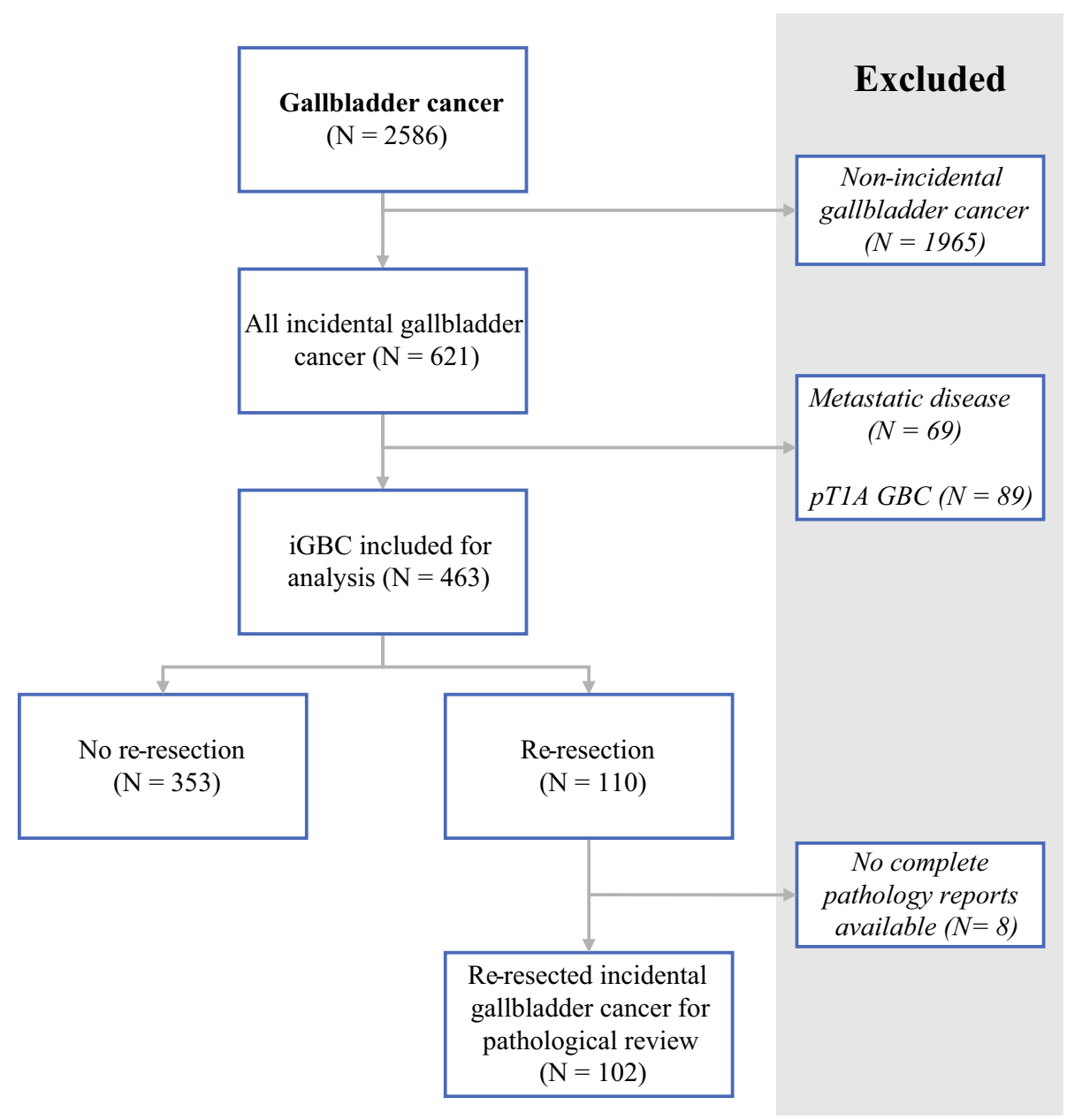

disease (Fig. 3b). Re-resection in T1b iGBC was not significantly associated with longer survival (median OS reresected $\mathrm{T} 1 \mathrm{~b}=56.0$ months vs. no resection $\mathrm{T} 1 \mathrm{~b}=60.0$ months; $p=0.705)$.

\section{Prognostic Factors and Survival After Re-resection}

In patients who received a re-resection and for whom complete pathology reports were available $(n=102)$, median OS was 56.3 months (95\% CI 32.3-80.2) in patients with tumor-free resection margins versus 18.0 months (95\% CI 13.1-23.0) in patients with tumorpositive resection margins in re-resection specimens $(p<0.001)$ (Fig. 4a). No significant survival difference was seen between patients who did and did not receive any form of liver resection (i.e. gallbladder bed, segmentectomy, or hemihepatectomy), neither across the entire cohort (50.0 vs. 52.6 months; $p=0.601)$ nor stratified according to $\mathrm{T}$ stage (data not shown). Median OS in patients without $\operatorname{RD}(N=66)$ in the re-resection specimen was not reached, versus 23.1 months (95\% CI 18.8-27.5) in patients in whom RD was present $(N=36$; $p<0.001)$ (Fig. 4b). No survival differences were found between different locations of RD; patients with RD in the liver only ( $n=13$ ) had a median OS of 22.9 months, versus 24.5 months in patients with $\mathrm{RD}$ in the lymph nodes alone ( $n=16)$ and 22.3 months in patients with RD in both liver and lymph nodes $(n=7)(p=0.257)$. In patients with $\mathrm{RD}$, no significant difference in median OS was found between patients with tumor-free resection margins (R0, $n=8$; median OS 24.5 months) in the re-resection specimen and patients in which re-resection margins were not clear (R1, $n=28$; median OS 16.1 months) ( $p=0.447)$.

On univariable screening, significant prognostic factors associated with worse outcome after re-resection were pT3 stage, irradical (R1/R2) resection margins after re-resection, perineural and lymphovascular invasion, and the presence of RD in the lymph nodes, cystic duct, and liver 
TABLE 1 Baseline patient and tumor characteristics

\begin{tabular}{lccr}
\hline & Re-resection $(N=110)$ & No re-resection $(N=353)$ & $p$ value \\
\hline Age, years [mean (range)] & $62.9(36-81)$ & $72.2(25-97)$ & $<\mathbf{0 . 0 0 1}$ \\
Sex, male & $33(30.0)$ & $93(26.3)$ & 0.452 \\
Tumor differentiation grade & & & \\
Well & $16(14.5)$ & $38(10.8)$ & \\
Moderately & $47(40.9)$ & $124(35.1)$ & \\
Poor & $22(20.0)$ & $101(28.6)$ & 0.079 \\
Unknown & $27(24.5)$ & $90(25.5)$ & \\
pT stage & & $47(13.3)$ & \\
T1b & $10(9.1)$ & $185(52.4)$ & \\
T2 & $74(67.3)$ & $90(25.5)$ & $\mathbf{0 . 0 0 1}$ \\
T3/T4 & $24(21.8)$ & $31(8.8)$ & \\
Tx & $2(1.8)$ & $132(37.4)$ & \\
pN stage & & $22(6.2)$ & \\
N0 & $22(20.0)$ & $199(56.4)$ & \\
N1-2 & $13(11.8)$ & $160(45.3)$ & \\
N $x$ & $75(68.2)$ & $95(26.9)$ & \\
Resection margin & & $98(27.8)$ & \\
R0 & $73(66.4)$ & & \\
R1/R2 & $32(29.1)$ & $(4.5)$ &
\end{tabular}

Data are expressed as $n(\%)$ unless otherwise specified

Bolded values indicate statistical significance $(P<0.005)$
(Table 2). In the multivariable Cox proportional hazards model, only the presence of RD in the lymph nodes (HR $2.35 ; p=0.005$ ) or liver (HR 5.54; $p<0.001$ ) remained significant prognostic factors (Table 2 ).

\section{Predictive Factors for Residual Disease}

On univariable screening, $\mathrm{N} 1$ disease, $\mathrm{T} 3$ disease, R1/R2 resection margins at primary cholecystectomy, lymphovascular invasion, and perineural invasion were predictive for RD in re-resected patients (Table 3). When entered into a multivariable model, only N1 (HR 23.0; $p=0.022$ ) and pT3 disease (HR 25.3; $p=0.003$ ) remained predictive of the presence of RD.

\section{DISCUSSION}

The present study demonstrates that re-resection was associated with increased survival in patients with $\mathrm{T} 2$ and $\mathrm{T} 3 \mathrm{iGBC}$, and that re-resection remained an independent favorable prognostic factor in multivariable analysis. In patients who underwent re-resection, RD was more often found in patients with a higher pT stage, and the presence of RD was the primary determinant of worse survival.
Although international guidelines recommend radical cholecystectomy for all iGBC patients, except those with $\mathrm{T} 1 \mathrm{a}$ disease, the management of $\mathrm{T} 1 \mathrm{~b}$ iGBC remains controversial. Results from the literature are conflicting; some studies do not report a survival benefit, ${ }^{22-24}$ whereas other series show an increase in 5-year survival of up to $30 \%$ after radical cholecystectomy. ${ }^{25,26}$ Interestingly, although a general survival benefit was shown across the entire reresected cohort, patients with T1b disease did not show superior survival after re-resection. Potentially, re-resection in $\mathrm{T} 1 \mathrm{~b}$ disease is not beneficial due to the low prevalence of $\mathrm{RD}$; only 1 of $10 \mathrm{~T} 1 \mathrm{~b}$ patients had RD. Another explanation might be that the extent of surgery in these patients was too small to provide a survival benefit; in all $\mathrm{T} 1 \mathrm{~b}$ patients, only a $1-2 \mathrm{~cm}$, non-anatomic wedge resection of the gallbladder bed was performed and median lymph node harvest was only 2 . This may have resulted in understaging and undertreatment and masked the potential benefit of radical cholecystectomy. Finally, our cohort was potentially too small to detect a significant difference in survival.

On the other hand, re-resection for T3 patients is currently not considered standard practice in the Dutch national guideline due to a lack of perceived benefit. ${ }^{27}$ In our cohort, median OS was 1 year in patients without reresection and 1.9 years in re-resected patients with $\mathrm{T} 3$ 
FIG. 2 Extent of resection and incidence of residual disease according to $\mathrm{T}$ stage

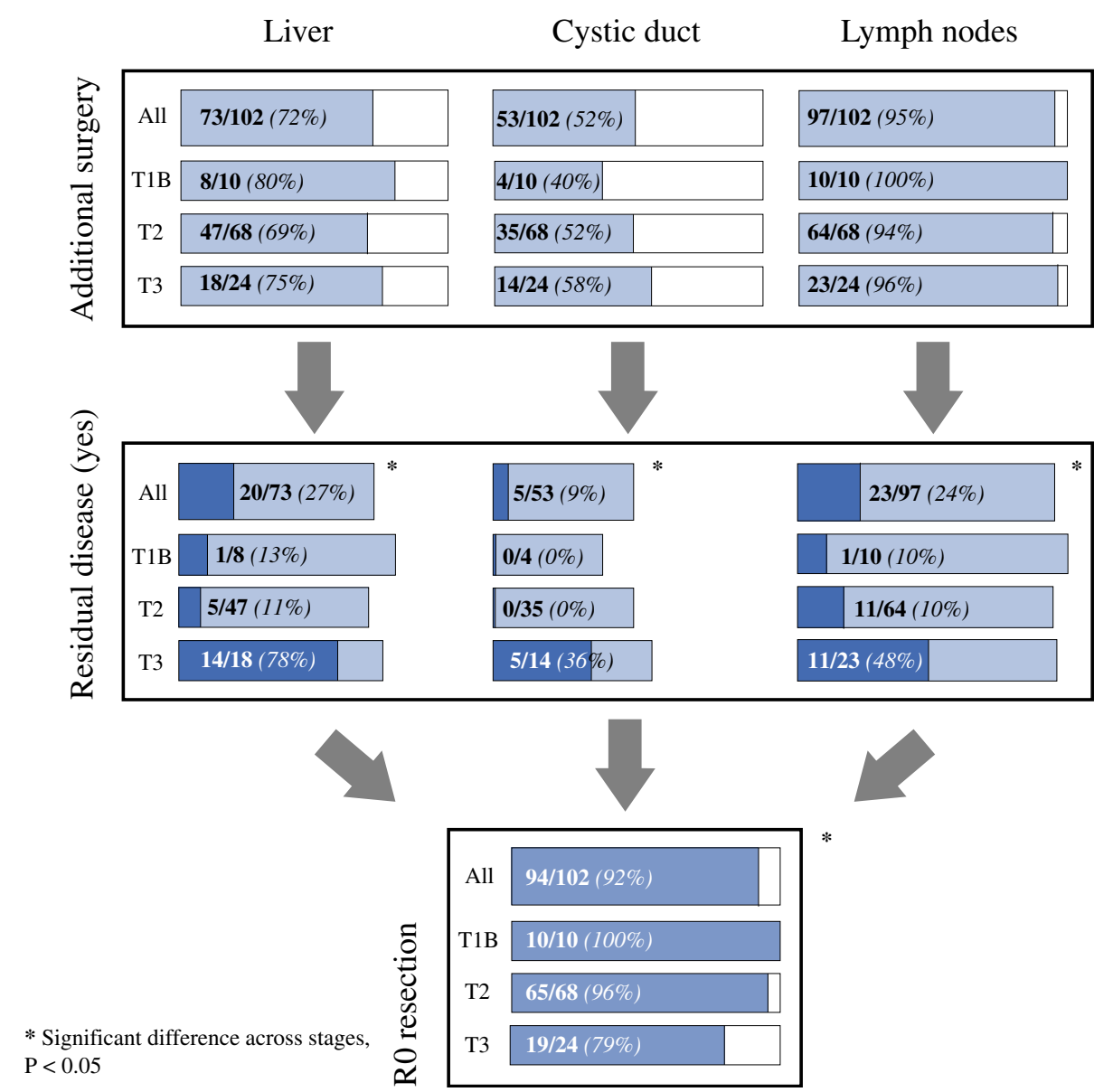

disease (landmark at 90 days). Four of 24 (17\%) patients with T3 disease had tumor-free margins at primary surgery. After re-resection, tumor-free margins were achieved in $19 / 24$ patients (79\%). Survival in GBC is primarily determined by the ability to achieve tumor-free margins $;^{10}$ therefore, the increased rate of R0 resections after re-resection is the most likely cause for the higher survival in reresected $\mathrm{T} 3$ disease.

International guidelines recommend re-resection for all patients with $\geq \mathrm{T} 1 \mathrm{~b}$ iGBC fit to undergo surgery within 4-8 weeks from the initial cholecystectomy. ${ }^{13,28}$ Worrisomely, in our cohort, only $23 \%$ of patients received a reresection and the median time interval between index surgery and re-resection was over 9 weeks. In a recent publication from Sweden, 121/201 (60\%) non-metastatic iGBC patients received a re-resection. ${ }^{24}$ In 27 (13\%) of all patients, a re-resection was not performed due to comorbidities. Another study included 218 iGBC patients and reresection was attempted in $188(86 \%)$ patients. ${ }^{16}$ Only 17
(8\%) patients did not undergo a re-resection due to low performance status. Unfortunately, due to the nature of our study, we were not able to assess the rationale for not performing a re-resection in our cohort; however, it is evident from other studies that comorbidities do not frequently preclude re-resection. Other factors such as physician unawareness of or ambiguity regarding the efficacy of re-resection may account for the low number of reresected patients in our cohort.

Moreover, our results show considerable practice variation regarding the extent of re-resection performed. International guidelines recommend gallbladder bed resection for all patients, as well as lymphadenectomy with a minimum count of six nodes. ${ }^{13}$ In our cohort, only $72 \%$ of patients received any form of liver resection and the median lymph node harvest was three. Evidently, guideline adherence is suboptimal and more extensive surgery than is currently performed is necessary to improve outcomes. 
FIG. 3 a Overall survival of patients with iGBC $(N=423$ after exclusion of follow-up of $<90$ days), by re-resection (yes, no). Log-rank $p<0.001$. b Overall survival of patients with $\mathrm{T} 2(N=243)$ and $\mathrm{T} 3 /$ $\mathrm{T} x(N=130)$ iGBC after exclusion of follow-up of $<90$ days, by re-resection. Log-rank $p<0.001$. $i G B C$ incidental gallbladder cancer

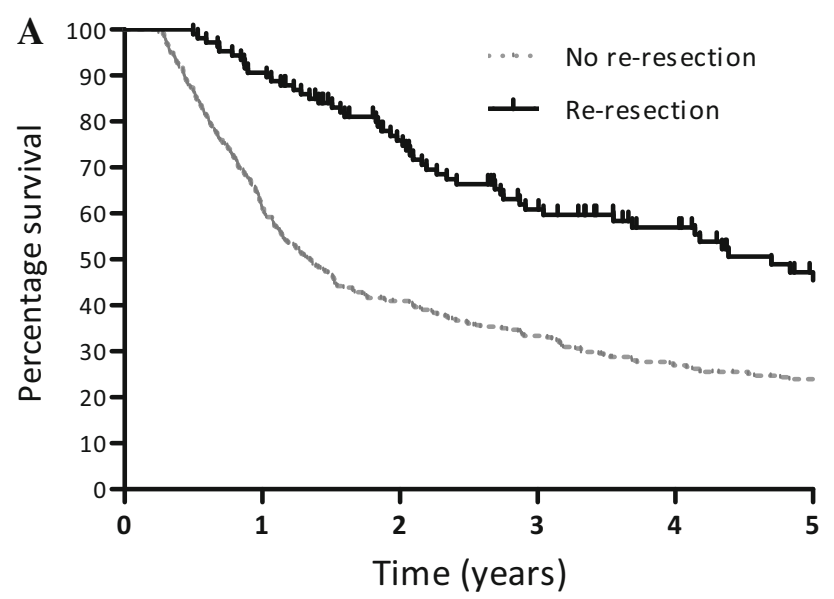

\begin{tabular}{llllllll}
\hline No re-resection & $\mathrm{N}$ & 316 & 195 & 127 & 99 & 75 & 62 \\
\hline Re-resection & $\mathrm{N}$ & 107 & 98 & 74 & 56 & 41 & 27 \\
\hline
\end{tabular}

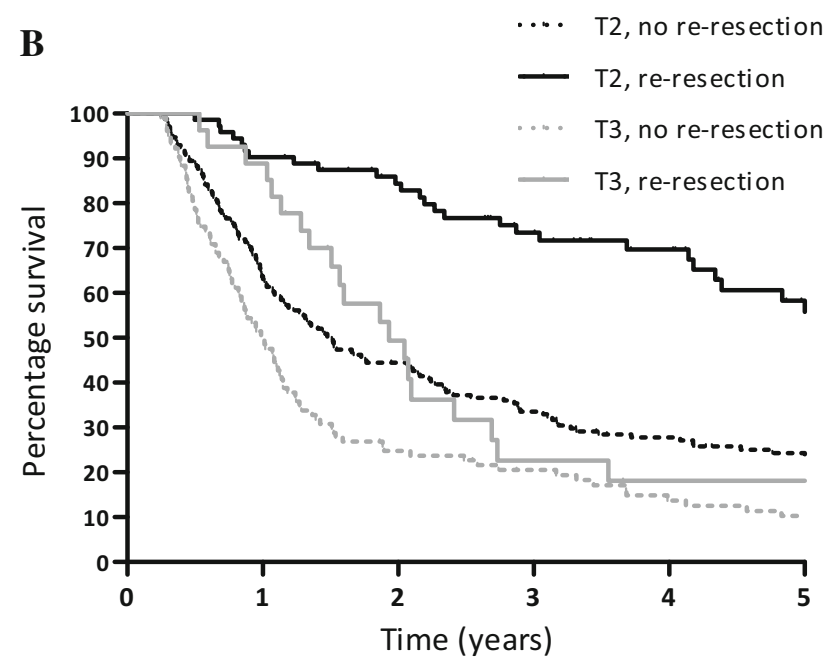

Additionally, our results raise concerns on the accuracy $\mathrm{RD}$ is mostly found in T3 disease and (regardless of site) is the primary determinant of survival after re-resection is in line with previously published literature.

The finding that RD is the primary determinant of survival raises questions about the value of re-resection in iGBC. The goal of re-resection is to clear the patient of residual local or regional disease and consequently improve survival. However, survival in patients in which $\mathrm{RD}$ is found is poor, even when resection margins are clear. Moreover, no significant survival difference was found between patients with RD who received R0 versus R1 reresection. This contradicts the notion that the increase in 
FIG. 4 Overall survival of patients with iGBC after reresection $(N=102)$, by a margin status and $\mathbf{b}$ residual disease. Log-rank $p<0.001$. $i G B C$ incidental gallbladder cancer, $R D$ residual disease

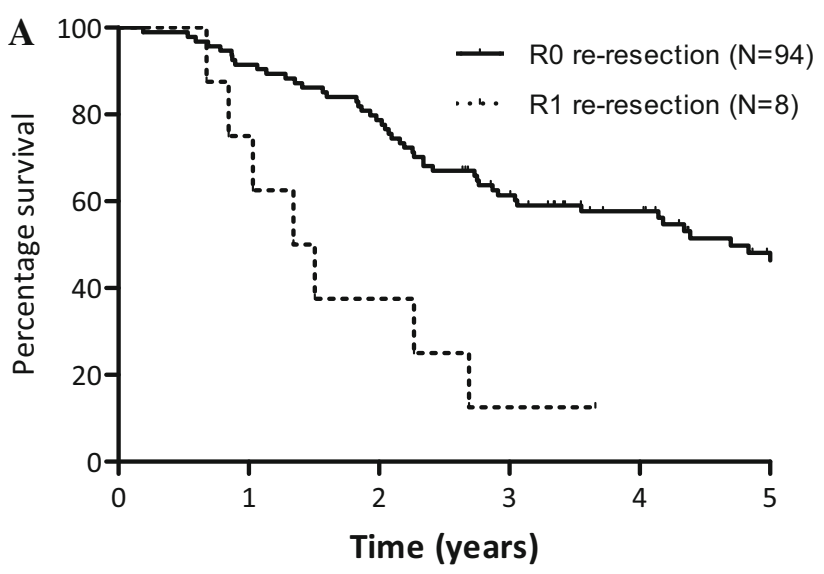

\begin{tabular}{llllllll}
\hline R0 & $\mathrm{N}$ & 94 & 87 & 75 & 55 & 42 & 28 \\
\hline R1 & $\mathrm{N}$ & 8 & 7 & 4 & 2 & 1 & 1 \\
\hline
\end{tabular}

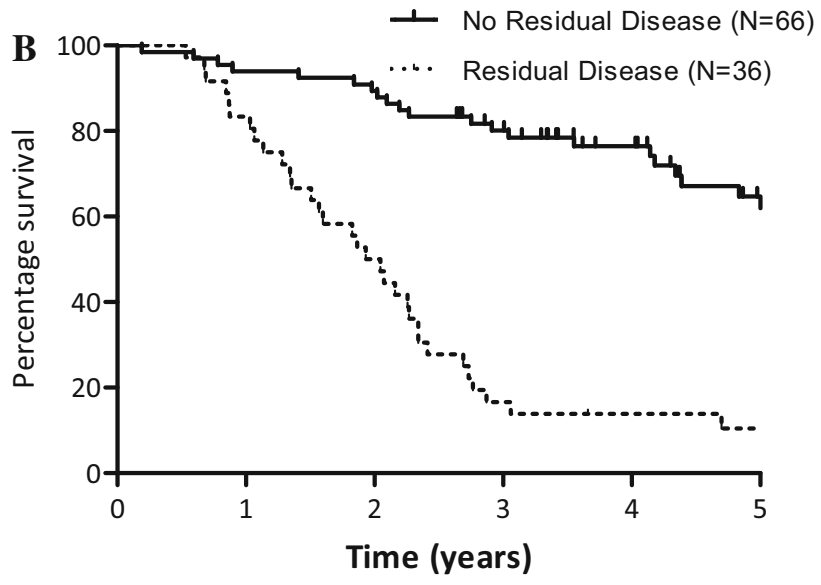

\begin{tabular}{llllllll}
\hline $\mathrm{RD}-$ & $\mathrm{N}$ & 66 & 63 & 60 & 50 & 38 & 25 \\
\hline $\mathrm{RD}+$ & $\mathrm{N}$ & 36 & 31 & 19 & 7 & 5 & 4 \\
\hline
\end{tabular}

survival seen after re-resection stems from complete tumor clearance. Potentially, re-resection is beneficial solely for patients in which only microscopic RD, undetected by the pathologist, is present. When macroscopic RD is found, the tumor may have already progressed beyond potential curation. The fact that survival between patients with different locations of RD did not differ suggests that the presence of $\mathrm{RD}$ acts as the clinical and prognostic equivalent of metastatic disease.

The fact that patients with RD appear unlikely to benefit from surgical treatment alone gives rise to novel clinical challenges. Although data are lacking, patients with iGBC may benefit from (neo)adjuvant chemotherapy, especially when RD is present. Predicting which patients are likely to have RD could be a useful tool to identify potential candidates for neoadjuvant treatment. Perineural and lymphovascular invasion, R1/R2 margins at initial cholecystectomy, tumor grade, and $\mathrm{pT}$ and $\mathrm{pN}$ stage were univariably associated with the presence of $\mathrm{RD}$ in our cohort. After multivariable analysis, only $\mathrm{pT}$ and $\mathrm{pN}$ stage appeared predictive for $\mathrm{RD}$, although CIs were wide. The other factors may have remained significant if more patients were included. Two studies with larger cohorts produced similar results; ${ }^{29,30}$ however, CIs were either not reported or were very wide, and $\mathrm{pN}$ stage was not included in their models. Future, larger cohorts are needed to further identify histopathological characteristics associated with RD.

This study has several limitations. Primarily, our results are sensitive to selection bias due to the retrospective study design. For example, improved survival after re-resection in T3 disease may very well be a result of treatment 
TABLE 2 Prognostic factors for survival after re-resection in patients with incidental gallbladder cancer $(N=102)$

\begin{tabular}{|c|c|c|c|c|c|c|}
\hline \multirow[t]{2}{*}{ Characteristic } & \multicolumn{3}{|c|}{ Univariable cox regression } & \multicolumn{3}{|c|}{ Multivariable cox regression } \\
\hline & HR & $95 \% \mathrm{CI}$ & $p$ value & HR & $95 \% \mathrm{CI}$ & $p$ value \\
\hline Age, years & 1.02 & $0.99-1.05$ & 0.156 & & & \\
\hline \multicolumn{7}{|l|}{ Pathological N stage } \\
\hline N0 & 1 & & & & & \\
\hline $\mathrm{N} 1 / \mathrm{N} 2$ & 0.72 & $0.38-1.35$ & 0.303 & & & \\
\hline $\mathrm{N} x$ & 1.38 & $0.80-2.37$ & 0.247 & & & \\
\hline \multicolumn{7}{|l|}{ Pathological T stage } \\
\hline $\mathrm{T} 1$ & 1 & & & c & & \\
\hline $\mathrm{T} 2$ & 1.42 & $0.50-4.01$ & 0.512 & c & & \\
\hline $\mathrm{T} 3 / \mathrm{T} x$ & 4.09 & $1.39-12.04$ & 0.011 & c & & \\
\hline \multicolumn{7}{|l|}{ Radicality re-resection } \\
\hline R0 & 1 & & & $\mathrm{c}$ & & \\
\hline $\mathrm{R} 1 / \mathrm{R} 2$ & 3.93 & $1.74-8.88$ & 0.001 & c & & \\
\hline \multicolumn{7}{|l|}{ Tumor differentiation grade } \\
\hline Well & 1 & & & & & \\
\hline Moderate & 0.81 & $0.37-1.78$ & 0.606 & & & \\
\hline Poor & 1.20 & $0.52-2.80$ & 0.668 & & & \\
\hline Unknown & 0.82 & $0.34-1.95$ & 0.648 & & & \\
\hline Residual disease, lymph node (yes) & 3.18 & $1.84-5.52$ & $<0.001$ & 2.35 & $1.30-4.23$ & 0.005 \\
\hline Residual disease, liver (yes) & 7.08 & $3.57-14.05$ & $<0.001$ & 5.54 & 2.70-11.37 & $<\mathbf{0 . 0 0 1}$ \\
\hline Residual disease, cystic duct (yes) & 5.82 & $2.17-15.57$ & $<0.001$ & c & & \\
\hline Lymphovascular invasion (yes) $^{\mathrm{a}}$ & 2.31 & $1.36-3.91$ & 0.002 & c & & \\
\hline Perineural invasion (yes) ${ }^{\mathrm{b}}$ & 1.86 & $1.06-3.27$ & 0.031 & c & & \\
\hline
\end{tabular}

All variables with $p<0.10$ on univariable analysis were entered into the multivariable model

Bolded values indicate statistical significance $(P<0.005)$

$H R$ hazard ratio, $C I$ confidence interval

${ }^{\mathrm{a}}$ Missing values in 10 cases

${ }^{\mathrm{b}}$ Missing values in 13 cases

${ }^{\mathrm{c}}$ Not significant during forward selection selection bias and immortal time bias rather than a potential therapeutic effect of re-resection. We attempted to address these biases by landmarking, multivariable analysis, and subgroup analysis in younger patients; however, some bias may still be present. Second, pathology reports were reviewed but no revision of the actual resection specimens was performed. Review by an expert hepatobiliary pathologist may have altered our results. Finally, survival according to $\mathrm{T}$ stage in non-re-resected patients may have been underestimated due to understaging.

A strength of this study is that our results are based on actual nationwide outcomes, and generalizability is therefore likely high. Moreover, our study is the only study that 
TABLE 3 Predictive factors for the presence of residual disease after re-resection in patients with incidental gallbladder cancer $(n=102)$

\begin{tabular}{|c|c|c|c|c|c|c|}
\hline \multirow[t]{2}{*}{ Characteristic } & \multicolumn{3}{|c|}{ Univariable logistic regression } & \multicolumn{3}{|c|}{ Multivariable logistic regression } \\
\hline & HR & $95 \% \mathrm{CI}$ & $p$ value & HR & $95 \% \mathrm{CI}$ & $p$ value \\
\hline \multicolumn{7}{|l|}{ Tumor differentiation grade } \\
\hline Well & 1 & & & c & & \\
\hline Moderate & 2.49 & $0.62-9.96$ & 0.197 & c & & \\
\hline Poor & 5.03 & $1.17-21.59$ & 0.030 & c & & \\
\hline Unknown & 1.56 & $0.26-9.47$ & 0.632 & c & & \\
\hline \multicolumn{7}{|l|}{ Pathological N stage } \\
\hline 0 & 1 & & & 1 & & \\
\hline $1 / 2$ & 25.00 & $2.36-264.80$ & 0.008 & 23.00 & $1.57-337.44$ & $0.022 *$ \\
\hline Unknown & 1.00 & $0.284-3.53$ & 1.000 & 1.053 & $0.14-4.18$ & 0.763 \\
\hline \multicolumn{7}{|l|}{ Pathological T stage } \\
\hline $\mathrm{T} 1$ & 1 & & & 1 & & \\
\hline $\mathrm{T} 2$ & 0.93 & $0.20-5.44$ & 0.966 & 1.22 & $0.18-8.52$ & 0.838 \\
\hline $\mathrm{T} 3 / \mathrm{T} x$ & 11.60 & $3.04-131.73$ & 0.002 & 25.33 & $2.98-215.69$ & $0.003 *$ \\
\hline Time since index surgery (days) & 1.00 & $0.99-1.01$ & 0.788 & & & \\
\hline \multicolumn{7}{|l|}{ Radicality primary resection } \\
\hline $\mathrm{R} 0$ & 1 & & & $\mathrm{c}$ & & \\
\hline $\mathrm{R} 1 / \mathrm{R} 2$ & 4.80 & $1.97-11.70$ & 0.001 & c & & \\
\hline Unknown & 1.14 & $0.11-11.87$ & 0.911 & c & & \\
\hline Socioeconomic status (deciles) & 1.03 & $0.88-1.19$ & 0.743 & & & \\
\hline \multicolumn{7}{|l|}{ Hospital of diagnosis } \\
\hline Community & 1 & & & & & \\
\hline Academic & 0.21 & $0.03-1.73$ & 0.146 & & & \\
\hline Lymphovascular invasion (yes) $^{\mathrm{a}}$ & 4.25 & $1.77-10.18$ & 0.001 & c & & \\
\hline Perineural invasion (yes) ${ }^{\mathrm{b}}$ & 3.18 & $1.25-8.09$ & 0.015 & c & & \\
\hline
\end{tabular}

All variables with $p<0.10$ on univariable analysis were entered into the multivariable model

${ }^{\mathrm{a}}$ Missing values in 10 cases

${ }^{\mathrm{b}}$ Missing values in 13 cases

${ }^{\mathrm{c}}$ Not significant during forward selection

$* p$ value $<0.005$

used landmark and stratification techniques when investigating the value of re-resection in iGBC, thus reducing the effects of the aforementioned biases.

\section{CONCLUSION}

There is substantial surgical undertreatment for iGBC in The Netherlands; re-resection is associated with improved survival in $\mathrm{T} 2$ and $\mathrm{T} 3 \mathrm{iGBC}$. The presence of RD is the main prognostic factor for survival after re-resection and can be predicted by $\mathrm{pT}$ and $\mathrm{pN}$ stage. Additional histopathological research is necessary to identify candidates most likely to benefit from additional surgery and possible neoadjuvant chemotherapy.

ACKNOWLEDGMENT The authors would like to thank Esther van den Broek (PALGA) for providing support in obtaining the pathology reports.
DISCLOSURES The authors declare that they have no conflict of interest.

OPEN ACCESS This article is distributed under the terms of the Creative Commons Attribution 4.0 International License (http://crea tivecommons.org/licenses/by/4.0/), which permits unrestricted use, distribution, and reproduction in any medium, provided you give appropriate credit to the original author(s) and the source, provide a link to the Creative Commons license, and indicate if changes were made.

\section{REFERENCES}

1. Are C, Ahmad H, Ravipati A, et al. Global epidemiological trends and variations in the burden of gallbladder cancer. $J$ Surg Oncol. 2017;115:580-90.

2. Butte JM, Matsuo K, Gonen M, et al. Gallbladder cancer: differences in presentation, surgical treatment, and survival in patients treated at centers in three countries. J Am Coll Surg. 2011;212:50-61. 
3. Lau CSM, Zywot A, Mahendraraj K, Chamberlain RS. Gallbladder carcinoma in the United States: a population based clinical outcomes study involving 22,343 patients from the surveillance, epidemiology, and end result database (1973-2013). HPB Surg. 2017;2017:1532835.

4. Hundal R, Shaffer EA. Gallbladder cancer: epidemiology and outcome. Clin Epidemiol. 2014;6:99-109.

5. Randi G, Franceschi S, La Vecchia C. Gallbladder cancer worldwide: geographical distribution and risk factors. Int $J$ Cancer. 2006;118:1591-602.

6. Gil L, de Aretxabala X, Lendoire J, et al. Incidental gallbladder cancer: how residual disease affects outcome in two referral HPB centers from South America. World J Surg. 2019;43:214-20.

7. Cavallaro A, Piccolo G, Panebianco V, et al. Incidental gallbladder cancer during laparoscopic cholecystectomy: managing an unexpected finding. World J Gastroenterol. 2012;18:4019-27.

8. Dorobisz T, Dorobisz K, Chabowski M, et al. Incidental gallbladder cancer after cholecystectomy: 1990 to 2014. Onco Targets Ther. 2016;9:4913-6.

9. Lundgren L, Muszynska C, Ros A, et al. Are incidental gallbladder cancers missed with a selective approach of gallbladder histology at cholecystectomy? World J Surg. 2018;42:1092-9.

10. Soreide K, Guest RV, Harrison EM, et al. Systematic review of management of incidental gallbladder cancer after cholecystectomy. Br J Surg. 2019;106:32-45.

11. Steinert R, Nestler G, Sagynaliev E, et al. Laparoscopic cholecystectomy and gallbladder cancer. $J$ Surg Oncol. 2006;93:682-9.

12. Ethun CG, Le N, Lopez-Aguiar AG, et al. Pathologic and prognostic implications of incidental versus nonincidental gallbladder cancer: a 10-institution study from the United States extrahepatic biliary malignancy consortium. Am Surg. 2017;83:679-86.

13. Aloia TA, Jarufe N, Javle M, et al. Gallbladder cancer: expert consensus statement. HPB (Oxford). 2015;17:681-90.

14. Pawlik TM, Gleisner AL, Vigano L, et al. Incidence of finding residual disease for incidental gallbladder carcinoma: implications for re-resection. J Gastrointest Surg. 2007;11:1478-86. (Discussion 1486-7).

15. Butte JM, Kingham TP, Gonen M, et al. Residual disease predicts outcomes after definitive resection for incidental gallbladder cancer. J Am Coll Surg. 2014;219:416-29.

16. Fuks D, Regimbeau JM, Le Treut YP, et al. Incidental gallbladder cancer by the AFC-GBC-2009 Study Group. World J Surg. 2011;35:1887-97.

17. von Elm E, Altman DG, Egger M, et al. The strengthening the reporting of observational studies in epidemiology (STROBE) statement: guidelines for reporting observational studies. Lancet. 2007;370:1453-7.
18. Edge SB, American Joint Committee on Cancer, American Cancer Society. AJCC cancer staging handbook: from the AJCC cancer staging manual. New York: Springer; 2010.

19. Casparie M, Tiebosch AT, Burger G, et al. Pathology databanking and biobanking in The Netherlands, a central role for PALGA, the nationwide histopathology and cytopathology data network and archive. Cell Oncol. 2007;29:19-24.

20. Rubin DB. Multiple imputation for nonresponse in surveys. New York: Wiley; 2004.

21. Mackinnon A. The use and reporting of multiple imputation in medical research: a review. J Intern Med. 2010;268:586-93.

22. Shirai Y, Yoshida K, Tsukada K, Muto T, Watanabe H. Early carcinoma of the gallbladder. Eur J Surg. 1992;158:545-8.

23. Wakai T, Shirai Y, Yokoyama N, et al. Early gallbladder carcinoma does not warrant radical resection. $B r \quad J \quad$ Surg. 2001;88:675-8.

24. Lundgren L, Muszynska C, Ros A, et al. Management of incidental gallbladder cancer in a national cohort. $\mathrm{Br} J$ Surg. 2019;106(9):1216-27.

25. Hari DM, Howard JH, Leung AM, et al. A 21-year analysis of stage I gallbladder carcinoma: is cholecystectomy alone adequate? HPB (Oxford). 2013;15:40-8.

26. Abramson MA, Pandharipande P, Ruan D, Gold JS, Whang EE. Radical resection for T1b gallbladder cancer: a decision analysis. HPB (Oxford). 2009;11:656-63.

27. Landelijke Werkgroep Gastro-intestinale tumoren (2013). Galweg-en galblaascarcinoom. Retrieved from https://www.onc oline.nl/galweg-en-galblaascarcinoom.

28. Ethun CG, Postlewait LM, Le N, et al. Association of optimal time interval to re-resection for incidental gallbladder cancer with overall survival: a multi-institution analysis from the US extrahepatic biliary malignancy consortium. JAMA Surg. 2017;152:143-9.

29. Creasy JM, Goldman DA, Gonen M, et al. Predicting residual disease in incidental gallbladder cancer: risk stratification for modified treatment strategies. $J$ Gastrointest Surg. 2017;21:1254-61.

30. Ethun CG, Postlewait LM, Le N, et al. A novel pathology-based preoperative risk score to predict locoregional residual and distant disease and survival for incidental gallbladder cancer: a 10-institution study from the U.S. extrahepatic biliary malignancy consortium. Ann Surg Oncol. 2017;24:1343-50.

Publisher's Note Springer Nature remains neutral with regard to jurisdictional claims in published maps and institutional affiliations. 